\title{
Novel insights into the insect trancriptome response to a natural DNA virus
}

\author{
Seanna J McTaggart ${ }^{1,2^{*}}$, Tidbury Hannah ${ }^{3}$, Stephen Bridgett ${ }^{4}$, Jennie $S$ Garbutt $^{1}$, Gaganjot Kaur ${ }^{5}$ and Mike Boots ${ }^{6}$
}

\begin{abstract}
Background: Little is known about invertebrate responses to DNA viruses. Here, we infect a commercially important pest moth species Plodia interpunctella with its naturally infecting DNA virus. We sequenced, assembled and annotated the complete transcriptome of the moth, and a partial transcriptome of the virus. We then tested for differential gene expression between moths that were exposed to the virus and controls.

Results: We found 51 genes that were differentially expressed in moths exposed to a DNA baculovirus compared to controls. Gene set enrichment analysis revealed that cuticle proteins were significantly overrepresented in this group of genes. Interestingly, 6 of the 7 differentially expressed cuticle proteins were downregulated, suggesting that baculoviruses are able to manipulate its host's response. In fact, an additional 29 of the 51 genes were also downregulated in exposed compared with control animals, including a gram-negative binding protein. In contrast, genes involved in transposable element movement were upregulated after infection.
\end{abstract}

Conclusions: We present the first experiment to measure genome-wide gene expression in an insect after infection with a natural DNA virus. Our results indicate that cuticle proteins might be key genes underpinning the response to DNA viruses. Furthermore, the large proportion of genes that were downregulated after viral exposure suggests that this virus is actively manipulating the insect immune response. Finally, it appears that transposable element activity might increase during viral invasion. Combined, these results provide much needed host candidate genes that respond to DNA viral invaders.

Keywords: Differential gene expression, DNA virus, de novo transcriptome assembly, host-pathogen interaction, RNA-Seq

\section{Background}

Hosts are constantly challenged by the ubiquitous presence of pathogens and understanding the genetic architecture of these interactions is critical to developing tools to prevent infection. Within insects, characterization of innate immune system genes and pathways has primarily focused on responses to bacterial and fungal pathogens [1]. In contrast, the genes that respond to viruses are less well understood, with the vast majority of research limited to work with RNA viruses [2]. Our understanding of insect immune responses to DNA viruses is poor and is a critical void that needs to be filled. Two studies that have

\footnotetext{
* Correspondence: smctagga@staffmail.ed.ac.uk

${ }^{1}$ Institute of Evolutionary Biology, School of Biological Sciences, Ashworth

Laboratories University of Edinburgh, Edinburgh EH9 3JT, UK

${ }^{2}$ Centre for Immunity, Infection and Evolution, School of Biological Sciences,

Ashworth Laboratories University of Edinburgh, Edinburgh EH9 3JT, UK

Full list of author information is available at the end of the article
}

quantified gene expression in Drosophila after exposure to a DNA virus (Invertebrate iridescent virus 6) demonstrated that the RNA interference (RNAi) pathway can be effective at removing DNA as well as RNA viruses, although how this occurs is unknown [3,4]. In addition, the evolutionarily conserved immune pathway JAK-STAT may play a role in both DNA and RNA viral defence, but only against a subset of viruses [4].

However, these two studies, like many of the functional studies elucidating the roles of invertebrate immune system genes, do not challenge the host with a natural pathogen. Since the immune response to a novel pathogen is unlikely be equivalent to the product of an antagonistic co-evolutionary relationship, it is imperative to also probe the immune response in naturally-occurring host-parasite systems. Additionally, these studies were carried out in a single species of Drosophila and therefore the generality of their findings are unclear. Finally, comparative genomics 
of fully sequenced insect genomes reveals that not all immune system genes are present in all taxonomic groups. For example, the pea aphid, Acyrthosiphon pisum, does not have key genes involved in recognition and signalling in the IMD immune pathway [5]. Thus, in order to build a comprehensive understanding of innate immune systems, it is important to survey a wide variety of taxonomic groups exposed to different pathogen types. Given our lack of understanding of the immune responses to DNA viruses, it is particularly important to examine natural DNA viral infections of non-model hosts.

To this end, we exposed a moth species, Plodia interpunctella, to its naturally infecting DNA virus Plodia interpunctella Granulosis Virus (PiGV). P. interpunctella, the Indian meal moth, is a major pest of stored dry food products around the world, causing significant economic losses [6]. We sequenced the complete transcriptomes of exposed and control moths using RNA-Seq (Illumina), and assembled them using and comparing two different commonly used assemblers (SOAPdenovo-Trans [7] and Trinity [8]). The chosen transcriptome assembly of the moth and a partial transcriptome assembly of the virus are available as a public resource at http://afterparty.bio.ed.ac.uk/. Finally, we characterized the virally-induced transcriptome of the moth, therefore adding much needed information on the genetic architecture of insect- DNA virus interactions.

\section{Results}

\section{De novo transcriptome assembly assessments}

After filtering for high quality sequences, a total of $488,010,769$ sequences (per sample average $=27,111,709 \mathrm{sd}$ $5,923,264$ ) were used to construct four assemblies using alternate methods. All samples were used to construct each assembly. Overall, the assemblies constructed with Trinity had a greater maximum contig length than SOAPdenovo-
Trans (62,936 bases versus 46,673 bases), while the SOAPdenovo-Trans assembly had a larger median contig length than the Trinity assembly (428 bases versus 393 bases) (Table 1). Both of the Trinity assemblies had many more contigs than either of the SOAPdenovo-Trans assemblies (135,990 contigs versus 82,753 contigs), which were longer (32,900 contigs $>=1 \mathrm{~kb}$ versus 20,725 contigs $>=1 \mathrm{~kb})$. Likewise, many more bases were included in the Trinity assemblies compared to the SOAPdenovo-Trans assemblies (140,975,202 compared to $85,762,961$ bases, respectively).

There were very minor differences in the number of reads that mapped to each of the assemblies (Table 2). Over $90 \%$ of the reads mapped back to each of the four assemblies, with the most (98\%) mapping to the Trinity assembly (Edge threshold $=0.16$ ). They all had equivalent numbers of uniquely mapping reads (which was the only category of mapped reads that was considered in the analysis of differential expression) ranging from $68-74 \%$.

Prior to searching each of the transcriptomes for ultraconserved orthologs (UCO), we used USearch [9] to collapse contigs that differed by less than $3 \%$ sequence divergence from the chosen Trinity assembly. This resulted in $\sim 9 \%$ reduction in the number of contigs. More of the UCO were recovered with the Trinity assemblies compared to the SOAPdenovo-Trans assemblies (Table 3), and more genes were only found once within the Trinity versus the SOAPdenovo-Trans assemblies. The Trinity assembly with the parameter edge threshold $=0.16$ recovered slightly more of the UCOs only once, compared to the assembly with an edge threshold of 0.05 , and thus the former was chosen for the differential expression analysis. After analysis with the EviGene pipeline, the contig set was reduced to $\mathrm{N}=18,475$. However, to ensure that all putative transcripts were made publically available, the full (i.e. uncollapsed) transcriptome was used as the input to afterParty [10] and

Table 1 Transcriptome assembly statistics

\begin{tabular}{|c|c|c|c|c|}
\hline & SOAPtransden & & Trinity & \\
\hline & kmer size $=\mathbf{2 5}$ & kmer size $=31$ & Edge threshold $=0.05$ & Edge threshold $=0.16$ \\
\hline Max contig length & 46,673 & 46,631 & 62,936 & 62,936 \\
\hline Mean contig length & 1,053 & 1,036 & 1,037 & 905 \\
\hline Standard deviation of contig length & 1,595 & 1,559 & 1,736 & 1,542 \\
\hline Median contig length & 424 & 428 & 393 & 375 \\
\hline N50 contig length & 2,539 & 2,452 & 2,617 & 2,125 \\
\hline Number of contigs & 80,425 & 82,753 & 135,990 & 127,665 \\
\hline Number of contigs $>=1 \mathrm{~kb}$ & 20,249 & 20,725 & 32,900 & 26,818 \\
\hline Number of contigs in N50 & 8,933 & 9,330 & 14,198 & 13,689 \\
\hline Number of bases in all contigs & $84,708,481$ & $85,762,961$ & $140,975,202$ & $115,544,987$ \\
\hline Number of bases in contigs $>=1 \mathrm{~kb}$ & $60,770,939$ & $60,961,055$ & $101,519,875$ & $77,125,576$ \\
\hline GC Content of contigs & $38.82 \%$ & $38.95 \%$ & $39.35 \%$ & $38.96 \%$ \\
\hline
\end{tabular}


Table 2 Mapping statistics

\begin{tabular}{|c|c|c|c|c|c|c|c|c|}
\hline & \multicolumn{4}{|c|}{ SOAPtransdenovo } & \multicolumn{4}{|l|}{ Trinity } \\
\hline & kmer size $=\mathbf{2 5}$ & $\%$ & kmer size=31 & $\%$ & Edge threshold $=0.05$ & $\%$ & Edge threshold $=0.16$ & $\%$ \\
\hline Total \# of fragments & $376,604,607$ & & $376,604,607$ & & $376,604,607$ & & $376,604,607$ & \\
\hline Total \# of mapping fragments & $345,067,834$ & 91.63 & $356,499,243$ & 94.66 & $351,206,829$ & 93.26 & $368,474,636$ & 97.84 \\
\hline \# of chr in reference & 80,425 & & 82,753 & & 135,990 & & 127,665 & \\
\hline \multicolumn{9}{|l|}{ Uniquely mapping pairs } \\
\hline Concordant & $236,768,017$ & 68.61 & $252,262,424$ & 70.76 & $240,006,041$ & 68.34 & $260,088,477$ & 70.59 \\
\hline Halfmapping & $10,181,639$ & 2.95 & $10,833,441$ & 3.04 & $4,222,926$ & 1.20 & $3,128,004$ & 0.85 \\
\hline unpaired & $1,674,474$ & 0.49 & $2,975,582$ & 0.83 & 758,404 & 0.22 & 925,244 & 0.25 \\
\hline \multicolumn{9}{|l|}{ Multiply mapping pairs } \\
\hline Concordant & $93,559,753$ & 27.11 & $87,279,976$ & 24.48 & $103,451,332$ & 29.46 & $100,980,778$ & 27.41 \\
\hline halfmapping & $2,102,511$ & 0.61 & $2,270,699$ & 0.64 & $1,287,909$ & 0.37 & $1,710,051$ & 0.46 \\
\hline unpaired & 581,342 & 0.17 & 611,289 & 0.17 & $1,240,292$ & 0.35 & $1,256,968$ & 0.34 \\
\hline \multicolumn{9}{|l|}{ Translocated read pairs } \\
\hline concordant & 0 & & 0 & & 0 & & 0 & \\
\hline Halfmapping & 0 & & 0 & & 0 & & 0 & \\
\hline unpaired & 0 & & 0 & & 0 & & 0 & \\
\hline \multicolumn{9}{|l|}{ Others } \\
\hline paired multimapping & 35,508 & 0.01 & 33,876 & 0.01 & 48,569 & 0.01 & 53,351 & 0.01 \\
\hline paired unique inverted & 116,861 & 0.03 & 84,905 & 0.02 & 65,638 & 0.02 & 71,371 & 0.02 \\
\hline paired unique long & 25,618 & 0.01 & 50,100 & 0.01 & 7,667 & 0.00 & 8,165 & 0.00 \\
\hline paired unique scr & 22,111 & 0.01 & 96,951 & 0.03 & 118,051 & 0.03 & 252,227 & 0.07 \\
\hline No mapping & $31,536,773$ & 9.14 & $20,105,364$ & 5.64 & $25,397,778$ & 7.23 & $8,129,971$ & 2.21 \\
\hline
\end{tabular}

the reduced transcriptome is available as a subset of these data.

\section{Functional annotation}

A total of 5396 (29\%) contigs were not homologous with any sequences present in the NBCI non-redundant database. Of the remaining 13,079 contigs that had a BLAST result, 9917 were not functionally classified. The remaining 3162 contigs were successfully annotated with GO terms. From the functionally annotated contigs, putative genes from the RNAi, IMD, Toll and JNK pathways were identified, although not all genes within these pathways were present (Table 4). The biological process categories (level 2) that contained the highest percentage of annotated genes were (1) cellular process (24\%) and (2) metabolic process (22\%). These two categories also represent the two largest sets of genes in the transcriptomes derived from the whole body of the pod borer, Maruca vitrata [11], as well as that of the midgut of the insect Manduca sexta [12] (Figure 1). However, a chi-square test shows that the distributions of GO terms between the three species' transcriptomes

Table 3 The number of recovered conserved orthologs found in each of the four de novo Plodia interpunctella transcriptomes

\begin{tabular}{|c|c|c|c|c|c|c|c|c|}
\hline \multirow[b]{2}{*}{ Number of copies } & \multicolumn{2}{|c|}{ SOAP kmer25 } & \multicolumn{2}{|c|}{ SOAP kmer31 } & \multicolumn{2}{|c|}{ Trinity 0.05} & \multicolumn{2}{|c|}{ Trinity 0.16} \\
\hline & Original & USearch & Original & USearch & Original & USearch & Original & USearch \\
\hline 0 & 28 & 28 & 26 & 26 & 21 & 21 & 21 & 21 \\
\hline 1 & 153 & 180 & 174 & 205 & 175 & 209 & 191 & 216 \\
\hline 2 & 104 & 103 & 91 & 89 & 83 & 90 & 85 & 90 \\
\hline 3 & 37 & 25 & 35 & 18 & 27 & 16 & 23 & 13 \\
\hline 4 & 16 & 13 & 17 & 12 & 19 & 11 & 15 & 8 \\
\hline 5 or more & 19 & 8 & 14 & 7 & 32 & 10 & 22 & 9 \\
\hline
\end{tabular}

The search was conducted on $\mathrm{N}=357$ ultra-conserved orthologs. A count is given for the orginal transcriptome build and for the build after clustering with USearch. 
Table 4 Presence or absence of immune related genes in lepidopteral Plodia interpunctella

\begin{tabular}{|c|c|c|c|}
\hline Gene & Pathway & Elicited by & Present \\
\hline Dicer & RNAi & viruses & yes \\
\hline R2D2 & RNAi & viruses & yes \\
\hline Argonaut & RNAi & viruses & yes \\
\hline Aubergine & RNAi & viruses & yes \\
\hline vig & RNAi & viruses & No \\
\hline Armi & RNAi & viruses & yes \\
\hline Drosha & RNAi & viruses & yes \\
\hline PGRP & IMD & Bacteria & yes \\
\hline IMD & IMD & Bacteria & No \\
\hline dFADD & IMD & Bacteria & No \\
\hline Dredd & IMD & Bacteria & No \\
\hline dTAK & IMD & Bacteria & No \\
\hline IKKy & IMD & Bacteria & yes \\
\hline IKKb & IMD & Bacteria & yes \\
\hline Relish & IMD & Bacteria & yes \\
\hline Sick & IMD & Bacteria & No \\
\hline Dnr1 & IMD & Bacteria & No \\
\hline JNK & JNK & Bacteria & yes \\
\hline Нер & JNK & Bacteria & Yes \\
\hline Bsk & JNK & Bacteria & No \\
\hline Nimrod & receptors & & No \\
\hline DSCAM & receptors & & yes \\
\hline Hemes & receptors & & No \\
\hline GNBP & PAMP & Bacteria & yes \\
\hline Persephone & Toll & Fungi, Bacteria & No \\
\hline serine proesases & Toll & Bacteria & Yes \\
\hline Spatzle & Toll & Bacteria & Yes \\
\hline Toll & Toll & Bacteria & yes \\
\hline Pelle & Toll & Bacteria & yes \\
\hline MyD88 & Toll & Bacteria & yes \\
\hline Tube & Toll & Bacteria & No \\
\hline Cactus & Toll & Bacteria & yes \\
\hline Dorsal & Toll & Bacteria & yes \\
\hline Dif & Toll & Bacteria & No \\
\hline prophenoloxidase & Toll & Bacteria & Yes \\
\hline antimicrobial peptides & Toll,IMD/JNK & Bacteria & yes \\
\hline Domeless & JAKJSTAT & viruses & No \\
\hline JAK & JAK/STAT & viruses & Yes \\
\hline STAT & JAK/STAT & viruses & Yes \\
\hline TEP & JAK/STAT & viruses & Yes \\
\hline
\end{tabular}

All putative genes can be found at http://afterparty.bio.ed.ac.uk/study/show/ 2194070. $(\mathrm{p}<0.001)$ are different. Additionally, fewer GO terms were identified in the $M$. sexta data set than in P. interpunctella, however, this is likely because the transcriptome of $M$. sexta was derived from a specialized tissue (the midgut).

A total of 27 contigs spanning 95,556 nucleotides were likely of PiGV origin. A dot plot against Plutella xylostella granulovirus (NCBI reference sequence NC_002593.1), suggests that this assembly covers about $44 \%$ of the viral genome (Figure 2). This contig set is also available at the afterParty website (http://afterparty.bio.ed.ac.uk). Surprisingly, some of the genes recovered include genes expressed in late and very late infection stages, such as those involved in viral replication (DNA polymerase, DNA ligase, and a helicase) and transmission (envelope fusion protein and envelope fusion protein) [13] as well as a chitinase.

\section{Infection outcome and differential expression}

On average, $77.6 \%(\mathrm{sd}=7 \%)$ of the larvae that were exposed to the viral solution became infected. No larvae that were exposed to the control treatment were infected. Fifty-one genes were significantly differentially expressed 24 hours after exposure to PiGV, the majority $(\mathrm{N}=36)$ of which were downregulated (Figure 3). These 51 genes have many different functions, including one canonical immune system gene (a homolog of the gramnegative binding protein (GNBP) Osiris), 7 cuticle proteins, a juvenile-hormone binding protein, 5 genes potentially involved in transposition and 13 genes of unknown function. Gene set enrichment analysis determined that only the cuticle proteins were enriched in this data set $(\mathrm{FDR}=1.8 \mathrm{E}-2)$. Interestingly, not all genes of the same putative function were regulated in the same way: six of the cuticle proteins were virally downregulated while one of them was virally up-regulated.

One of the 51 differentially expressed genes was not of the host origin. Indeed, its closest homolog was a putative proteobacteria. This gene was very lowly expressed in virally exposed larvae and absent in controls. Additionally, 3 of the differentially expressed genes have no known similarity to any other proteins, thus it was not possible to determine whether they were of host, viral or some other origin.

\section{q-RT-PCR}

We analysed the expression of comp623_c0_seq1 (cuticle protein) and comp2004_c0_seq1 (GNBP) using comparative $C_{\mathrm{T}}\left(\Delta \Delta \mathrm{C}_{\mathrm{T}}\right)$ qPCR. The expression of both genes was significantly lower in moths that were exposed to the virus (Figure 4, note the log scale; comp623_c0_seq1 $t_{1,4}=6.34, p=0.003$; comp2004_c0_seq1 $t_{1,4}=3.55$, $\mathrm{p}=0.02)$. 


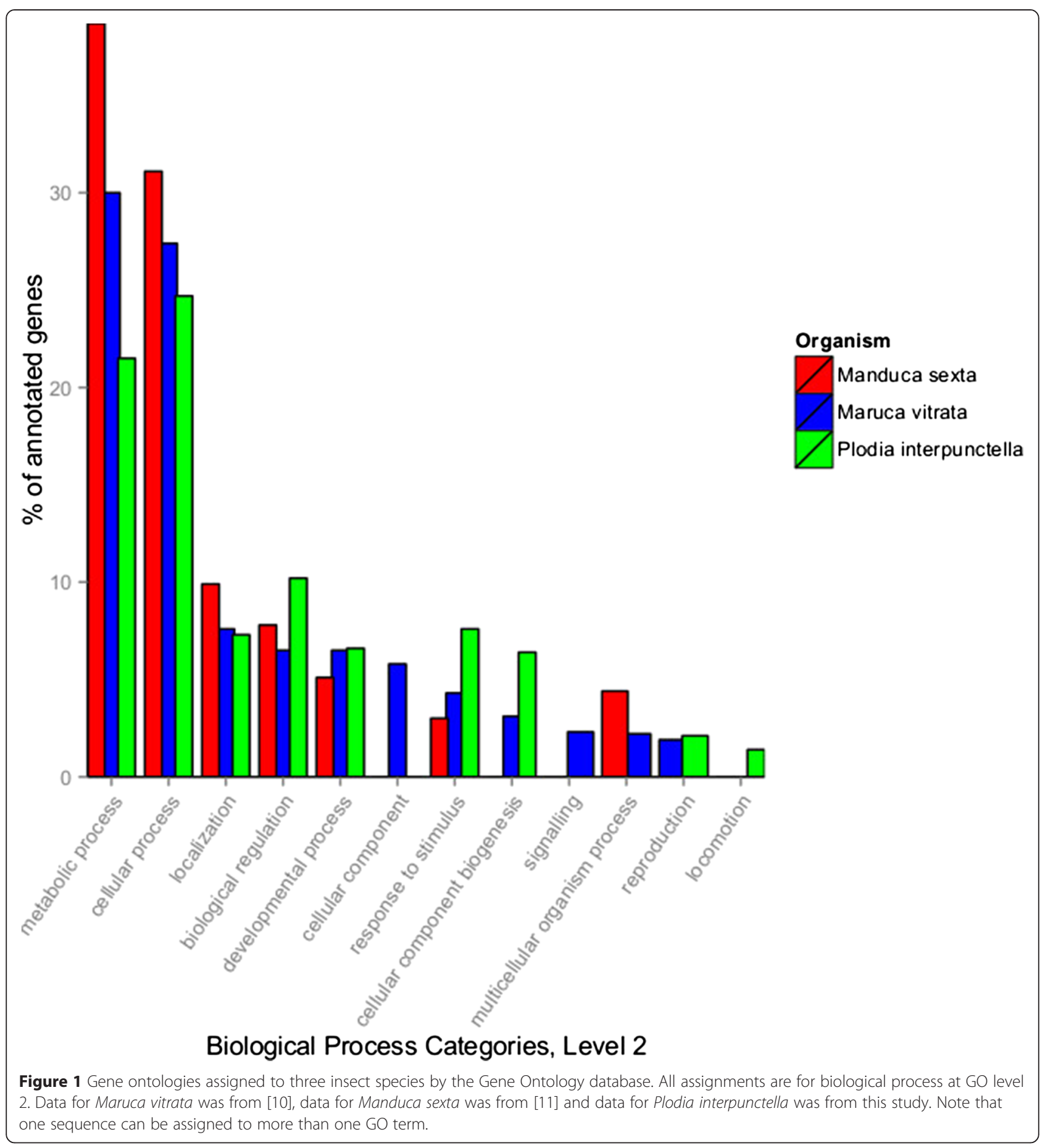

\section{Discussion}

Assembly and functional annotation

Two de novo transcriptomes were assembled from each of SOAPdenovotrans and Trinity. Overall, the Trinity assemblies contained more and longer contigs than the SOAP assemblies, and seemed to have slightly better coverage of highly conserved, single copy orthologous genes. In addition to recovering $95 \%$ of the ultra-conserved orthologs ( $60 \%$ of which were present as a single copy), gene candidates were identified from all canonical immune gene pathways (e.g. RNAi, Jak/STAT, Toll, IMD), supporting the relative completeness of the assembly. As expected for biological samples that were not controlled for life-history stage, experimental conditions or tissue type, when broken down into functional categories through the use of GO terms, the P. interpunctella 


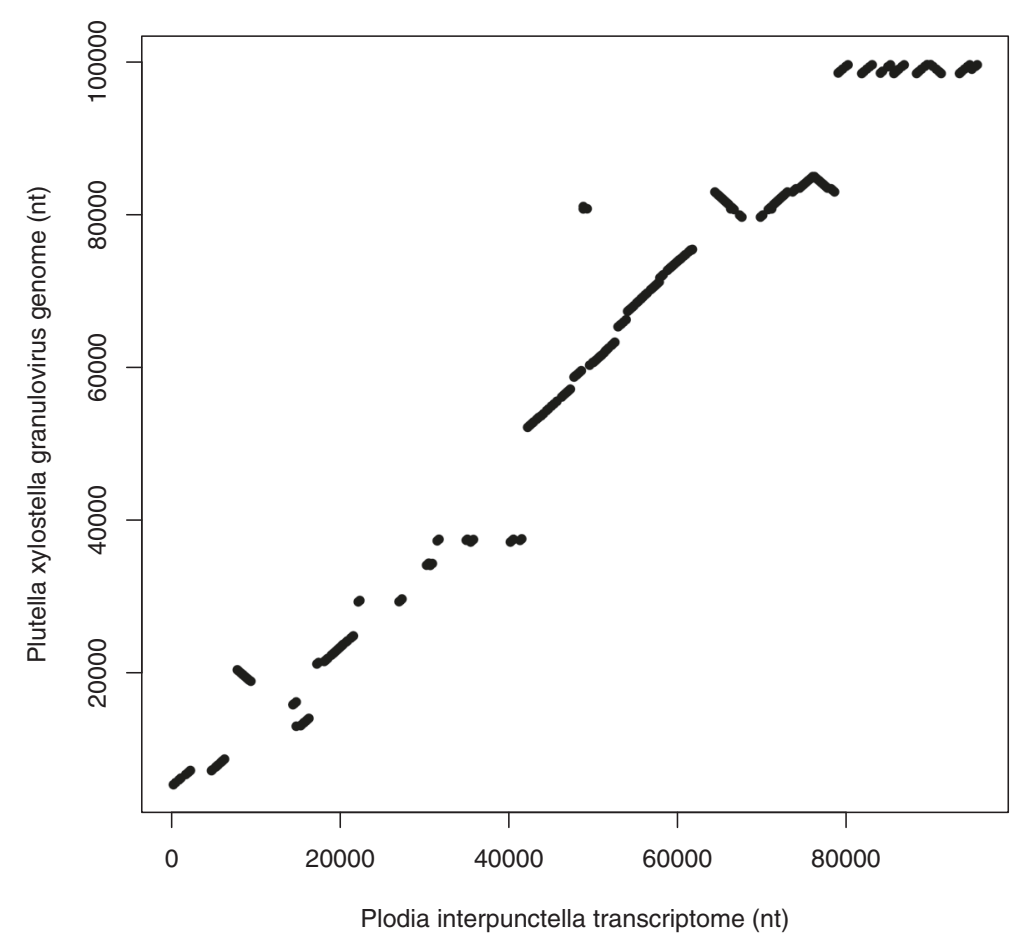

Figure 2 Nucleotide sequence similarity dot plot of the de novo transcriptome of Plodia interpunctella granulovirus (this study) versus the assembled Plutella xylostella granulovirus genome (NCBI reference sequence NC_002593.1). Nucleotide identity between the two sequences is represented by a dot. This analysis suggests that the current $P$. interpunctella granulovirus transcriptome assembly is approximately $44 \%$ complete.

de novo assembly differs from other lepidopteran transcriptomes (Figure 1).

\section{Differential expression}

Forty-seven $P$. interpunctella genes were differentially expressed 24 hours after exposure to PiGV compared to exposure to a control solution. The observed changes in expression could have several mechanistic origins, including defence, tolerance and repair. The differentially expressed genes were enriched for cuticle proteins $(\mathrm{N}=7), 6$ of which were virally downregulated and 1 was upregulated. To our knowledge, cuticle proteins have not been implicated previously from studies examining mRNA levels in cell cultures after infection with a baculovirus [14]. Cuticle proteins could play a role in defence in at least the following three ways. First, cuticle proteins form a large proportion of the peritrophic membrane (PM). The PM lines the gut and provides the first line of defence against ingested pathogens, such as PiGV and has been strongly implicated in antiviral defence $[15,16]$. For example, the PM of more susceptible velvetbean caterpillars, Anticarsia gemmatalis had a lower chitin content and provided a less efficient barrier against its baculovirus (AgMNPV) than more resistant larvae [15]. Furthermore, changes in the peritrophic membrane are correlated with changes in the risk of pathogen infection. For example, the thickness of the PM is also well known in Anopheles mosquitoes to increase after the ingestion of a blood meal, which is the primary source of infective pathogens [16].

The change in expression of cuticle proteins in P. interpunctella could reflect similar processes. Generally, cuticle proteins in the peritrophic membrane have a distinctive molecular signature, which is not present in any of the significantly differentially expressed cuticle proteins identified. However, since studies characterizing PMs have only been conducted in a limited number of species, which are not closely related to the lepidopteran $P$. interpunctella, it is possible that the molecular signature is too divergent to recognize. Furthermore, not all proteins of the PM have been characterized. For example, [17] recently identified a new PM protein in the meadow moth that is able to bind chitin, but does not contain the conserved binding domain. Secondly, expression of cuticle proteins has been monitored in insects and been shown to change when moulting takes place [18]. In Plodia, moulting includes shedding the gut lining, to which the PiGV particle may attach prior to penetration into the haemolymph. Thus, the differentially expressed cuticle proteins might correspond to Plodia moulting. Finally, the cuticle proteins may directly inhibit viral replication. For example, [19] demonstrated that a mosquito cuticle protein (AAEL011045) binds to a viral envelope and thus inhibits infection. Furthermore, they discovered that this protein was downregulated in virally exposed mosquitoes, raising the possibility 

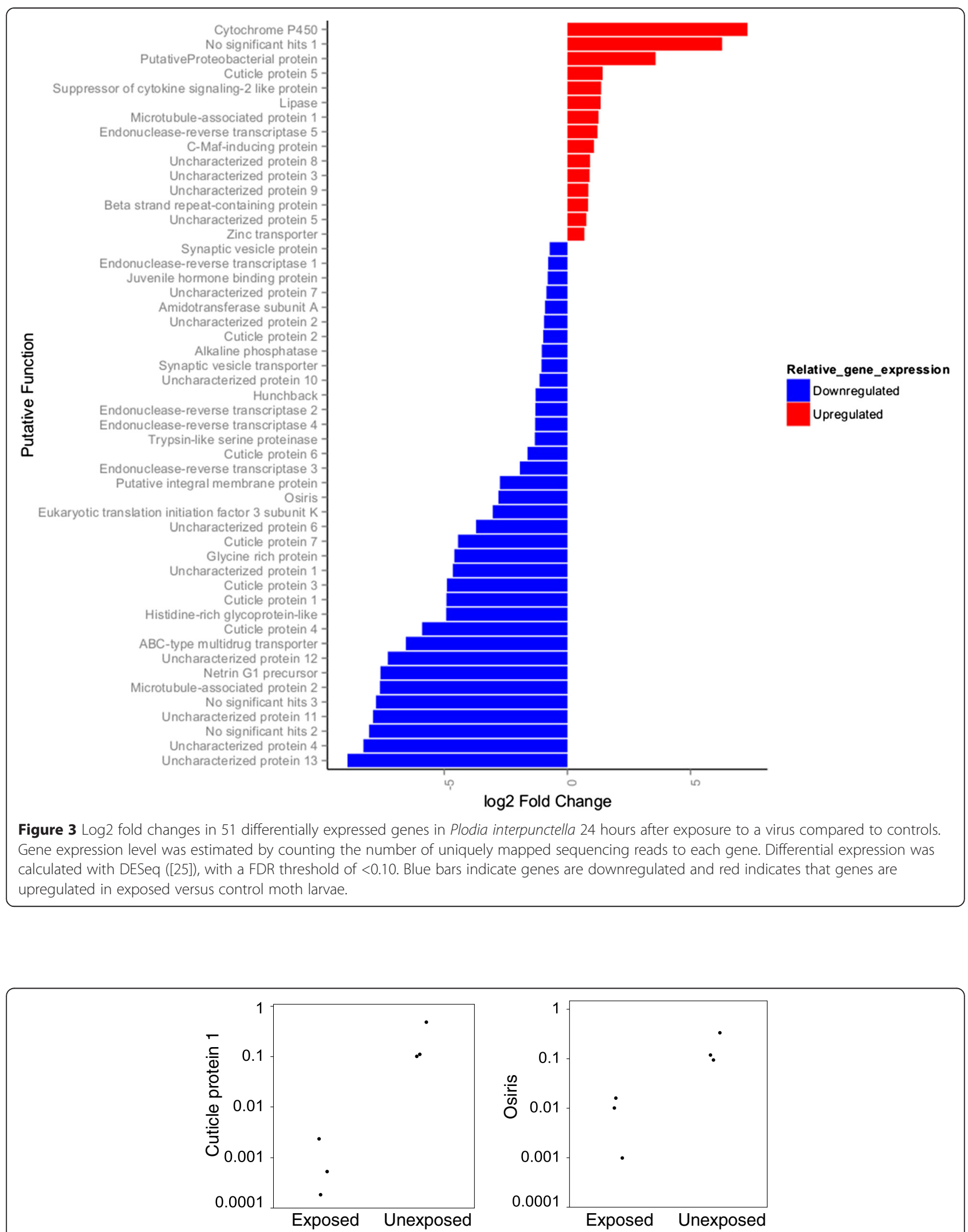

Figure 4 Confirmation of expression using q-RT-PCR. Expression of comp623_c0_seq1 (cuticle protein 1) and comp2004_c0_seq1 (Osiris (a putative GNBP)) relative to actin (comp24_c0_seq1) in the two infection treatments. 
that the virus was actively suppressing the expression of this molecule.

In order to discover if the degree of sequence identity amongst the 7 differentially expressed cuticle-proteins in this study could be used to explain their opposing pattern of regulation (i.e. up versus down), we aligned them with mosquito cuticle protein AAEL011045 in BioEdit v.7.2.4 [20] and built a neighbour-joining tree using CLUSTAL v1.2.0 [21] (Figure 5). This phylogenetic analysis shows that the seven moth genes cluster into three major clades; two clades of three genes each and a singleton. The singleton is the only cuticle protein that is upregulated after viral exposure. The moth genes in the clade containing the mosquito cuticle protein AAEL011045 are all candidate immune system genes that may be able to bind to the envelope of PiGV and thus inhibit its infectivity.

Two of the most up-regulated genes after viral exposure are a reverse transcriptase and a transposase. This suggests that within 24 hours of exposure to a virus, transposable elements (TE) activity, or 'jumping, is switched on (there were no reads from the control samples that mapped to either of these two genes). Supporting this hypothesis, a study that detected differential expression in hemocytes of the moth larvae Heliothis virescens after infection with Helicoverpa zea single nucleopolyhedrovirus found many retrotransposons to be upregulated [22]. A recent study in Drosophila showed that fragments of a virus were reversetranscribed, producing truncated versions of the virus, which were processed by the RNAi machinery resulting in a reduction in the amount of active virus in the cells [23]. Due to the increase in transposase activity found in this system, it will be interesting to test if there is a connection between TE activity and the control of a viral infection in this system as is the case in Drosophila.

Of all of the differentially expressed genes, 36 (70\%) were downregulated. The prevalence of down-regulation suggests that PiGV may directly or indirectly exert a considerable inhibitory effect on the host immune response. Indeed, late in infection, some studies in cell culture have documented a global downregulation of host genes (e.g. [24]). Similar effects of viral suppression on host immunity have been documented in other in vivo

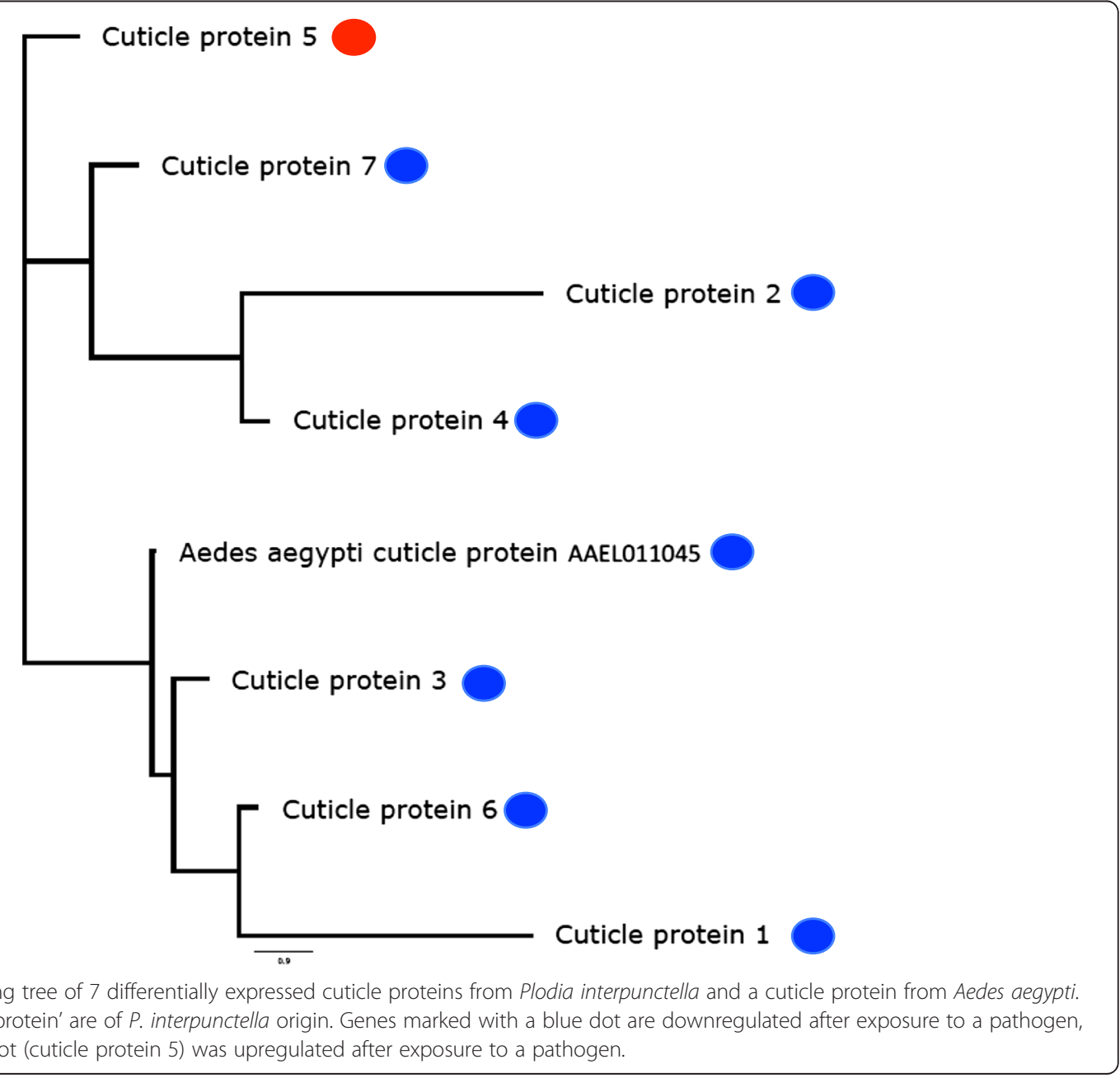


insect/viral systems (Aedes aegypti infected with Denguevirus [19,25], West Nile Virus and Yellow Fever Virus [19]). Indeed, in our differentially expressed data set, the only canonical immune system gene, a gram-negative binding protein (GNBP), is downregulated in virally-exposed larvae compared to control larvae. GNBPs are intricately involved activating the Toll pathway, and are upregulated after exposure to bacterial pathogens. The fact that the GNBP identified in this experiment is downregulated suggests that the virus may be suppressing the TOLL pathway. This potential for a trade-off between the immune response to bacteria versus viruses has been documented in mosquitoes, where the growth of $E$. coli was enhanced after viral infection with dengue virus (an RNA virus) in $A$. aegypti hosts [25]. They went on to show that viral interference with the host immune system resulted in a decreased production of antimicrobial peptides. Our transcriptome assembly contains several putative antimicrobial peptides, but none of them were differentially expressed after infection with the virus. However, our sampling time point was very early in the infection process, and thus sampling at more time points will be necessary to test if the viral infection has an effect on antimicrobial peptide activity.

The change in GNBP transcription may support the hypothesis that the innate immune response to a viral infection is not be restricted to the RNAi pathway, but instead additionally involves the Toll pathway. However, it is difficult to distinguish if the activation of different immune pathways is a direct or indirect result of the virus. For example, if the virus crossing the gut membrane results in a wound, naturally occurring gut microbes will also pass into the haemolymph and trigger an immune reaction. Our finding that one of the differentially expressed genes in the moth larvae was of bacterial origin is consistent with this hypothesis. Because we only assessed transcription very early in infection, it is not possible to determine if other members of the Toll pathway would also be differentially expressed.

Some of the genes that were determined to be differentially expressed are not functionally annotated $(\mathrm{N}=16$, $31 \%$ ). Many of these genes have high sequence similarity to other organisms, suggesting that functionally conserved, important putative immune, tolerance or repair genes have yet to be characterized in insects. Additionally, some of the genes had no known homology to other organisms, suggesting that there are also highly specific genes that are functionally uncharacterized. This study provides a solid foundation for choosing candidates for further functional characterization.

\section{Conclusions}

We have identified many candidate genes involved in the molecular response of a moth species to a naturally infecting DNA virus, a response for which we previously had a very limited understanding. Our results support a growing body of evidence that non-canonical immune system genes such as cuticle-proteins may play a significant role in the insect response to a range of pathogens. Additionally, transposition rates of mobile elements may be significantly altered during a viral attack.

Furthermore, this study represents the first comprehensive mRNA sequencing effort in this economically important pest species. The sequences from this study provide an important resource for studies of molecular genetics and functional genomics of Plodia interpunctella. For example, these data can be used to develop microarrays for gene expression analysis or to serve as a reference transcriptome for future RNA-seq experiments with $P$. interpunctella. Finally, we have also assembled a portion of the viral genome, which will allow molecular markers to be developed, which can, for example, aid in assessing the prevalence of this virus in natural populations.

\section{Methods}

\section{Larval culture, RNA extraction, library preparation and sequencing}

The host species used in our experiment was Plodia interpunctella, the Indian meal moth. All individuals were taken from a large outbred stock that has been maintained at the University of Sheffield for approximately 8 years and reared on a cereal-based diet under standard laboratory conditions $\left(27^{\circ} \mathrm{C} ; 16 \mathrm{~L}: 8 \mathrm{D}\right.$ cycle). The pathogen used was Plodia interpunctella Granulosis Virus (PiGV), a natural DNA virus of P. interpunctella. The virus is naturally transmitted through the ingestion of virus occlusion bodies either from the environment or through necropsy of infected individuals. Baculoviruses (BVs) are generally host-specific, obligate killing DNA viruses [26] with two phenotypes; the occluded virus, which consists of virions encased within a protein coat, and the budded virus. The initial site of virus exposure in the insect is the midgut where the alkaline conditions destroy the occlusion body resulting in the release of infectious virions. These virions may cross the peritrophic membrane into the epithelial cells and systemic infection is established when progeny BVs pass across the basal lamina into the haemocoel and infect secondary tissue such as the tracheal system and the fat body [27]. Our aim was to capture the change in mRNA levels during early infection, as immune responses can be swift and transient [28]. To this end, we chose to sample at 24 hours post infection, at which point enveloped virus nucleocapsids were found trapped in the connective tissue surrounding the midgut [29].

Transcriptome assemblies were constructed from $18 P$. interpunctella samples sequenced with RNA-Seq (Illumina) 100 base, pair-end reads on three HiSeq lanes (Illumina). The 18 samples came from two independent experiments, 
one of which $(\mathrm{N}=12)$ is described in a separate paper (McTaggart et al., in prep). Each sample consisted of 20 pooled individuals. Here, our aim was to characterize the transcriptomes of larvae, 24 hours after they had been exposed to viral dose of $\mathrm{LD}_{50}$. We set up six replicates in each of two treatments (viral exposed and control). In each replicate, $30 \mathrm{~F} 1$ generation adult moths were kept together with excess food. Prior to their viral exposure, 50 fourth instar larvae were removed from the pot, starved for 2 hours and then inoculated with the virus using a standard droplet feeding method [27]. The virus solution was prepared by centrifugation of infected $P$. interpunctella larvae, and diluted in blue food colouring and sucrose. The required dose was calculated from a dose response assay performed prior to the experiment.

We inoculated larvae with either a solution of purified PiGV to $\mathrm{LD}_{50}$ or with a control solution consisting of only sucrose and blue food colouring. Both treatments were orally administered with a standard oral droplet feeding protocol, and considered successful if the blue solution was visible in at least half of the gut length. Inoculated larvae were moved to individual wells of a 25-cell Petri dish containing ample food. Twenty-four hours later twenty larvae from each replicate were pooled and crushed in $1 \mathrm{ml}$ of Trizol (Life Technologies) and stored at $-20^{\circ} \mathrm{C}$ until RNA extraction. The remaining larvae $(\mathrm{N} \sim 30)$ were monitored for infection for 30 days. Due to financial constraints, only three of the six replicates from each treatment (i.e. six samples in total) were sequenced using RNA-Seq.

Immediately before RNA extraction, an additional 500ul of TRIzol (Ambion, Life Technologies) was added to each sample, and incubated for 5 minutes at room temperature. Two hundred ul of chloroform was added to each sample, shaken vigorously for 15-20 seconds and then centrifuged at $11600 \mathrm{rcf}$ for 15 minutes at $4^{\circ} \mathrm{C}$. The upper, aqueous phase was isolated and nucleic acids precipitated by adding 0.5 volumes of Absolute Ethanol, and inverting the tubes several times. This solution was used as the starting material for the RNAeasy (Qiagen) protocol, which was followed according to the manufacturer's instructions. The integrity of the resultant total RNA was confirmed on Bioanalyzer (Agilent RNA-nano reagents). RNA and DNA concentrations were determined with a Qubit fluorometer (Invitrogen QuantRNA), while the 260:280 ratio was assessed on a Nanodrop (ThermoScientific). For each sample, we subjected 5 ug of total RNA to one round of poly-A selection on oligo(dT) Serabeads. The resultant messenger RNA was fragmented to an average size of $100 \mathrm{bp}$ using divalent cations at $95^{\circ} \mathrm{C}$ for $5 \mathrm{~min}$ prepared following the manufacturer's recommended protocol (Illumina mRNAseq kits Cat no. RS-100-0801). First strand cDNA synthesis was carried out using Superscript III reverse transcriptase (Invitrogen) and $3 \mathrm{ug}$ random hexamer primers (Illumina) per sample as per the manufactures' instructions. Second strand cDNA synthesis and RNAseq samples were prepared according to the manufacturer's recommended protocol (Illumina). The fragment size and concentration of resultant libraries were assessed on a Qubit fluorometer (Invitrogen QuantRNA) and on a Bioanalyser High Sensitivity Chip (Invitrogen QuantRNA).

\section{Transcriptome assemblies}

Adapter sequences were trimmed from the raw reads using the program Scythe (https://github.com/vsbuffalo/ scythe). The program Sickle (https://github.com/najoshi/ sickle) was used to remove low quality bases, reads with N's, and sequences that were less than 50 bases long. SOAPdenovo-Trans [7] was used to build two assemblies with different $\mathrm{k}$-mer sizes $(\mathrm{kmer}=25$, $\mathrm{kmer}=30)$. Otherwise, default parameter settings were used. The assembler Trinity [8] was also run on the data twice, once with an edge threshold parameter of 0.05 and the other of 0.16. Default settings were used for all of the other parameters. Reads were mapped back to each assembly using GSNAP (http://research-pub.gene.com/gmap/). Reads thought to be due to PCR duplication were flagged with Picard (http://broadinstitute.github.io/picard/) and were not considered in any analyses. All contigs that had three or fewer reads mapping to it across all treatments were considered to be misassembled and removed.

In order to determine if any of the assembled contigs were of viral origin, the complete genome of Plutella xylostella granulovirus (NC_002593.1)(PxGV) was queried against the $P$. interpunctella transcriptome. Any contig that had a BLAST hit with an e-value of less than 1e-30 was considered to be viral. A dot-plot of these transcripts was made against the PxGV genome to estimate how much of the PiGV transcriptome had been recovered.

\section{Assembly assessment}

For the purposes of comparison, we assessed three basic parameters of each of the four assemblies: contig length, the number of contigs and the number of bases in the contigs. We also quantified the proportion of the sequence reads that mapped to each assembly using GSNAP. To test the completeness of the assemblies, we tested for the presence of highly conserved genes from other species in two ways. First, we built a BLAST database of each transcriptome that was queried with a previously published set of ultraconserved single-copy orthologs (UCOs) using tBLASTn. We counted how many times each gene was observed (with the expectation that each gene should be present only once) in each transcriptome. Similarly, we used the program CEGMA [30], which uses a different set of highly conserved single copy orthologs to query the data sets. The preferred assembly was then run through the Evidence Gene pipeline (http://arthropods.eugenes.org/ 
Table 5 q-RT-PCR primers

\begin{tabular}{lll}
\hline Gene & F primer $\mathbf{( 5}^{\prime}$ - $\left.\mathbf{3}^{\prime}\right)$ & R primer $\left(\mathbf{5}^{\prime}\right.$ - $\left.\mathbf{3}^{\prime}\right)$ \\
\hline comp6230_c0_seq1 (cuticle) & CGG CTG GAA CTG ATT GCT AC & GTG TGG GAT GGA TGA TTG TG \\
comp2004_c0_seq1 (GNBP) & GAT GCG ACA CTA GAA TAG CTT GG & ACC CAT CTC AAC TCG CCT AC \\
comp24_c0_seq1 (actin) & GAT CTG GCA CCA CAC CTT CT & GGT CAT CTT CTC CCT GTT GG \\
\hline
\end{tabular}

genes2/about/EvidentialGene_trassembly_pipe.html). This pipeline reduces redundancy in de novo assemblies by translating each contig in all six reading frames, selecting the longest open reading frame. Pairwise comparisons between all contigs eliminate all that have the same protein sequences.

\section{Functional annotation}

We used the afterParty (https://github.com/mojones/AfterParty2) interface to annotate all of our contigs using BLAST to the Uniref90 database [31]. Canonical immune system genes from the RNAi, Toll, IMD and JNK pathways were manually annotated within, and are accessible at, the afterParty $P$. interpunctella database (http://afterparty.bio. ed.ac.uk/study/show/2194070). Contigs with a BLAST hit to the query having an e-value hit greater than 1e-10, and covering at least $80 \%$ of the full-length transcripts (inferred based on homology) were considered to be valid candidate transcripts. All contigs were loaded into Blast2Go, annotated with the non-redundant database of NCBI, and GO terms were assigned when possible. Combined graphs of the entire transcriptome were constructed for Biological Process and Molecular Function (level 2). These results were compared with the two other lepidopteran species for which equivalent data was available, Maruca vitrata [11] and Manduca sexta, with a chi-square test [12].

\section{Differential expression of immune system genes}

The number of times each contig in the chosen transcriptome was observed in the sequence reads was calculated using HTSeq (http://www-huber.embl.de/users/ anders/HTSeq/doc/overview.html). Differential expression between $P$. interpunctella that were exposed to PiGV compared to controls was calculated using DESeq (version 1.9.4) [32]. All genes (contigs) with an FDR correction of less than 0.10 were considered to be significant.

\section{q-RT-PCR}

We analysed the expression of comp6230_c0_seq1 (cuticle protein) and comp2004_c0_seq1 (GNBP) using comparative $\mathrm{C}_{\mathrm{T}}\left(\Delta \Delta \mathrm{C}_{\mathrm{T}}\right)$ qPCR with actin as the internal control gene. Using the RNA samples prepared for RNASeq, we synthesised complementary DNA (cDNA) by first mixing $500 \mathrm{ng}$ total RNA with $500 \mathrm{ng}$ random hexadeoxynucleotides (Promega) and heating to $75^{\circ} \mathrm{C}$ for 10 minute. The samples were chilled on ice after which
200 u MMLV reverse transcriptase (Promega), $5 \mu \mathrm{l} 5 \mathrm{x}$ MMLV reverse transcriptase buffer, $1.25 \mu \mathrm{l}$ dNTPs (Promega; final concentration $0.5 \mathrm{mM}$ ) and $20 \mathrm{u}$ RNAsin RNase inhibitor (Promega) was added and the volume adjusted to $25 \mu \mathrm{l}$ with nuclease free water. Samples were incubated at $37^{\circ} \mathrm{C}$ for 60 minutes, $70^{\circ} \mathrm{C}$ for 15 minutes and then stored at $-20^{\circ} \mathrm{C}$.

PCR was carried out using a StepOnePlus ${ }^{\mathrm{TM}}$ Real-Time PCR System (Applied Biosystems) and Fast SYBR Green Master Mix (Applied Biosystems) to monitor doublestranded DNA synthesis in combination with ROX as a passive reference dye. PCR reactions were carried out in duplicate using $7.5 \mathrm{pmol}$ specific primers and approximately $5 \mathrm{ng}$ cDNA in a total volume of $15 \mu \mathrm{l}$. The thermal profile for amplification was as follows: $95^{\circ} \mathrm{C}$ for 2 minutes, followed by 40 cycles of $95^{\circ} \mathrm{C}$ for 10 seconds, $58^{\circ} \mathrm{C}$ for 30 seconds and $60^{\circ} \mathrm{C}$ for 30 seconds. Primer pairs were designed by us and tested by standard curve analysis (primer sequences are detailed in Table 5). Expression of comp6230_c0_seq1 and comp2004_c0_seq1 (relative to actin expression) was analysed using t-tests to ask whether the expression (log transformed) was affected by infection treatment.

\section{Availability of supporting data}

The RNA-seq sequencing reads used to construct the assemblies are available in the SRA repository, http://www. ebi.ac.uk/ena/data/view/PRJEB5332

The assembled transcriptomes (and subsets therein) are available at (http://afterparty.bio.ed.ac.uk/study/show/ 2194070)

\section{Abbreviations}

BVs: Baculoviruses, differentially expressed: differential expression; JAK-STAT: Janus kinase-Signal Transducer and Activator of Transcription; IMD: Immune deficient; PiGV: Plodia interpuntella granulosis virus; PxGV: Plutella xylostella granulovirus; UCO: Ultra-conserved orthologs; RNAi: RNA interference; GO: Gene ontology; GNBP: Gram-negative binding protein; TE: Transposable element; FDR: False discovery rate; PM: Peritrophic membrane.

\section{Competing interests}

The authors declare that they have no competing interests.

\section{Authors' contributions}

HT participated in the design of the study and carried out the infection experiments. SB and GK constructed the four transcriptome assemblies. JSG performed the real-time qPCR. SJM conceived of the study, prepared the samples for sequencing, did all analyses and wrote the paper. MB provided statistical advice and helped edit the manuscript. All authors read and approved the final manuscript. 


\section{Acknowledgements}

The authors gratefully acknowledge Edinburgh Genomics for RNA-Seq library preparation and sequencing, and Martin Jones for early access to the afterParty database. This work was funded by a NERC Biomolecular Analysis Facility (NBAF) small grant to SJM.

\section{Author details}

${ }^{1}$ Institute of Evolutionary Biology, School of Biological Sciences, Ashworth Laboratories University of Edinburgh, Edinburgh EH9 3JT, UK. ${ }^{2}$ Centre for Immunity, Infection and Evolution, School of Biological Sciences, Ashworth Laboratories University of Edinburgh, Edinburgh EH9 3JT, UK. ${ }^{3}$ Centre for Environment, Fisheries and Aquaculture Science, The Nothe, Barrack Road, Weymouth, Dorset DT4 8UB, UK. ${ }^{4}$ Edinburgh Genomics, Institute of Evolutionary Biology, School of Biological Sciences, Ashworth Laboratories University of Edinburgh, Edinburgh EH9 3JT, UK. ${ }^{5}$ The Centre for Applied Genomics, The Hospital for Sick Children, Toronto, ON M5G 1X8, Canada. ${ }^{6}$ Daphne du Maurier Centre for Ecology and Conservation, College of Life and Environmental Sciences, University of Exeter, Cornwall Campus, Cornwall TR10 9EZ, UK

\section{Received: 19 February 2014 Accepted: 27 March 2015}

\section{Published online: 17 April 2015}

\section{References}

1. Kounatidis I, Ligoxygakis P. Drosophila as a model system to unravel the layers of innate immunity to infection. Open Biol. 2012. doi:10.1098/ rsob. 120075 .

2. Kingsolver MB, Huang Z, Hardy RW. Insect antiviral innate immunity: Pathways, effectors, and connections. J Mol Biol. 2013. doi:10.1016/j. jmb.2013.10.006

3. Bronkhorst AW, van Cleef KW, Vodovar N, Ince IA, Blanc H, Vlak JM, et al. The DNA virus Invertebrate iridescent virus 6 is a target of the Drosophila RNAi machinery. Proc Natl Acad Sci Usa. 2012. doi:10.1073/pnas.1207213109.

4. Kemp C, Mueller S, Goto A, Barbier V, Paro S, Bonnay F, et al. Broad RNA Interference-Mediated Antiviral Immunity and Virus-Specific Inducible Responses in Drosophila. J Immunol. 2013. doi:10.4049/jimmunol.1102486.

5. Gerardo NM, Altincicek B, Anselme C, Atamian H, Barribeau SM, de Vos M, et al. Immunity and other defenses in pea aphids, Acyrthosiphon pisum. Genome Biol. 2010. doi:10.1186/gb-2010-11-2-r21.

6. Phillips TW, Berbert RC, Cuperus GW. Encyclopedia of Food Science and Technology. 2nd ed. New York: Wiley Inc; 2000. p. 2690-701.

7. Xie Y, Wu G, Tang J, Luo R, Patterson J, Liu S, et al. SOAPdenovo-Trans: De novo transcriptome assembly with short RNA-Seq reads. Bioinformatics. 2013. doi: 10.1093/bioinformatics/btu07.

8. Grabherr MG, Haas BJ, Yassour M, Levin JZ, Thompson DA, Amit I, et al. Full-length transcriptome assembly from RNA-Seq data without a reference genome. Nat Biotechnol. 2011. doi:10.1038/nbt.1883.

9. Edgar RC. Search and clustering orders of magnitude faster than BLAST. Bioinformatics. 2010. doi:10.1093/bioinformatics/btq461.

10. Jones $\mathrm{M}$, Blaxter $\mathrm{M}$. afterParty: turning raw transcriptomes into permanent resources. BMC Bioinf. 2013. doi:10.1186/1471-2105-14-301.

11. Margam VM, Coates BS, Bayles DO, Hellmich RL, Agunbiade T, Seufferheld MJ, et al. Transcriptome sequencing, and rapid development and application of SNP markers for the Legume Pod Borer Maruca vitrata (Lepidoptera: Crambidae). PLoS One. 2011. doi:10.1371/journal. pone.0021388.

12. Pauchet $Y$, Wilkinson $P$, Vogel H, Nelson DR, Reynolds SE, Heckel DG, et al. Pyrosequencing the Manduca sexta larval midgut transcriptome: messages for digestion, detoxification and defence. Insect Mol Biol. 2010. doi:10.1111/j.1365-2583.2009.00936.x.

13. Rohrmann GF. Baculovirus structural proteins. J Gen Virol. 1992 doi:10.1099/0022-1317-73-4-749.

14. Nguyen Q, Nielsen LK, Reid S. Genome scale transcriptomics of baculovirus-insect interactions. Viruses. 2013. doi:10.3390/v5112721.

15. Levy SM, Falleiros AMF, Moscardi F, Gregório EA. The role of peritrophic membrane in the resistance of Anticarsia gemmatalis larvae (Lepidoptera: Noctuidae) during the infection by its nucleopolyhedrovirus (AgMNPV). Arthropod Struct Dev. 2011. doi:10.1016/j.asd.2011.05.003.

16. Kato N, Mueller CR, Fuchs JF, McElroy K, Wessely V, Higgs S, et al. Evaluation of the function of a type I peritrophic matrix as a physical barrier for midgut epithelium invasion by mosquito-borne pathogens in Aedes aegypti. Vector Borne Zoonotic Dis. 2008. doi:10.1089/vbz.2007.0270.

17. Yin J, Wei Z-J, Li K-B, Cao Y-Z, Guo W. Identification and molecular characterization of a new member of the peritrophic membrane proteins from the meadow moth, loxostege sticticalis. Int J Biol Sci. 2010 doi:10.7150/ijbs.6.491.

18. Okamoto S, Futahashi R, Kojima T, Mita K, Fujiwara H. Catalogue of epidermal genes: genes expressed in the epidermis during larval molt of the silkworm Bombyx mori. BMC Genomics. 2008. doi:10.1186/1471-2164-9-396.

19. Colpitts TM, Cox J, Vanlandingham DL, Feitosa FM, Cheng G, Kurscheid S, et al. Alterations in the Aedes aegypti transcriptome during infection with West Nile, dengue and yellow fever viruses. Plos Pathog. 2011. doi:10.1371/journal.ppat.1002189.

20. Hall TA. BioEdit: a user-friendly biological sequence alignment editor and analysis program for Windows 95/98/NT. Nucleic Acids Symp Ser. 1999;41:95-8.

21. Larkin MA, Blackshields G, Brown NP, Chenna R, McGettigan PA, McWilliam H, et al. ClustalW and ClustalX version 2. Bioinformatics. 2007;23:2947-8.

22. Breitenbach JE, Shelby KS, Popham HJR. Baculovirus induced transcripts in hemocytes from the larvae of Heliothis virescens. Viruses. 2011. doi:10.3390/v3112047.

23. Goic B, Vodovar N, Mondotte JA, Monot C, Frangeul L, Blanc H, et al. RNAmediated interference and reverse transcription control the persistence of RNA viruses in the insect model Drosophila. Nat Immunol. 2013. doi:10.1038/ni.2542.

24. Salem TZ, Zhang F, Thiem SM. Comprehensive analysis of host gene expression in Autographa californica nucleopolyhedrovirus-infected Spodoptera frigiperda cells. Virology. 2011. doi:10.1016/j.virol.2011.01.006

25. Sim S, Dimopoulos G. Dengue Virus Inhibits Immune Responses in Aedes aegypti Cells. PLoS One. 2010. doi:10.1371/journal.pone.0010678.

26. Blissard GW, Rohrmann GF. Baculovirus diversity and molecular biology. Annu Rev Entomol. 1990;35:127-55.

27. Boots M, Begon M. Trade-offs with resistance to a granulosis-virus in the Indian meal moth, examined by a laboratory evolution experiment. Funct Ecol. 1993;7:528-34.

28. Hoffman JA, Reichhart J-M, Hetru C. Innate immunity in higher insects. Curr Opin Immunol. 1996;8:8-13.

29. Begon M, Daud KBH, Young P, Howells RE. The invasion and replication of a granulosis virus in the indian meal moth, Plodia interpunctella: an electron microscope study. J Invertebr Pathol. 1993;61:281-95.

30. Parra G, Bradnam K, Korf I. CEGMA: a pipeline to accurately annotate core genes in eukaryotic genomes. Bioinformatics. 2007;23:1061-7.

31. Suzek BE, Huang H, McGarvey P, Mazumder R, Wu CH. UniRef: comprehensive and non-redundant UniProt reference clusters. Bioinformatics. 2007;23:1282-8.

32. Anders $\mathrm{S}, \mathrm{Huber}$ W. Differential expression analysis for sequence count data. Genome Biol. 2010. doi:10.1186/gb-2010-11-10-r106.

\section{Submit your next manuscript to BioMed Central and take full advantage of:}

- Convenient online submission

- Thorough peer review

- No space constraints or color figure charges

- Immediate publication on acceptance

- Inclusion in PubMed, CAS, Scopus and Google Scholar

- Research which is freely available for redistribution 